\title{
PESQUISA DE OOCISTOS DE Isospora spp. EM PASSERIFORMES CRIADOS EM CATIVEIRO
}

\author{
DeUVÂNia CARVALHo da SILVA ${ }^{1}$, CAMILA GUARIZ HOMEM ${ }^{1}$, ALEX AKIRA NAKAMURA ${ }^{1}$, VALÉRIA CRISTINA DA \\ SILVA ${ }^{2}$, MARCELO VASCONCELOS MEIRELES ${ }^{3}$
}

${ }^{1}$ Pós Graduandos da Faculdade de Medicina Veterinária da Universidade Estadual Paulista, Araçatuba, SP, Brasil.

${ }^{2}$ Graduanda da Faculdade de Medicina Veterinária da Universidade Estadual Paulista, Araçatuba, SP, Brasil.

${ }^{3}$ Professor Doutor da Faculdade de Medicina Veterinária da Universidade Estadual Paulista, Araçatuba, SP, Brasil. meirelesmv@uol.com.br

\section{RESUMO}

O presente estudo teve como objetivo pesquisar, em longo prazo, a presença de oocistos de Isospora spp. em várias espécies de passeriformes, naturalmente infectadas, criadas em cativeiro. Foram colhidas 289 amostras em dois criatórios de passeriformes, onde houve comprovação prévia de infecção por Isospora, nos quais havia alojamento de várias espécies de passeriformes adultos. As amostras foram colhidas de forma individual, com periodicidade mensal, por 13 meses, purificadas em solução de Sheather e examinadas por microscopia. Das 289 amostras, $159(55,02 \%)$ apresentaram positividade para oocistos de Isospora e 130 (44,98\%) foram negativas. $\mathrm{Na}$ maioria das aves analisadas foi observada eliminação de oocistos, em pequena quantidade, intermitente e por período prolongado. Apesar de todas as aves apresentarem oocistos de Isospora nas fezes pelo menos uma vez, em um período de 13 meses, as aves não apresentaram isosporose clínica. Os resultados observados neste experimento fornecem dados para o controle da isosporose em passeriformes criados em cativeiro. As decisões sobre a realização de tratamento profilático ou curativo, assim como sobre medidas higiênico-sanitárias a serem adotadas devem levar em consideração não somente a presença de parasito em fezes, mas também a intensidade de eliminação de oocistos, ssim como a avaliação do manejo higiênico sanitário e nutricional e a presença de sinais clínicos e/ou de mortalidade.

PALAVRAS-CHAVE: amostras de fezes; aves; Isospora spp.; oocistos.

\section{SCREENING FOR Isospora spp. OOCYSTS IN PASSERIFORMES RAISED IN CAPTIVITY}

\section{ABSTRACT}

This study aimed to research the long-term shedding of Isospora spp. oocysts in several species of passerines naturally infected and kept in captivity. Two hundred and eighty-nine fecal samples were collected from two flocks with previous diagnosis of isosporosis, in which several adult passerine species were raised. Samples were collected individually, monthly, for 13 months, purified in Sheather's sugar solution and examined using microscopy. Of the 289 samples, 159 (55.02\%) were positive for Isospora spp. oocysts and 130 (44.98\%) were negative. Most of the birds analyzed shed oocysts in small quantity (score 1), intermittently and for a long period. Despite the occurrence of Isospora infection, the 
birds that were analysed showed no clinical isosporosis. The results of this research provide data for the control of isosporosis in passerines raised in captivity. The decisions about performing prophylactic or curative treatment, as well as decisions related to hygiene and sanitary measures must take

KEYWORDS: birds; fecal samples; Isospora spp., oocysts.

\section{INTRODUÇÃO}

Várias espécies de aves da ordem Passeriformes são criadas em cativeiro no Brasil, seja como animais de estimação, em zoológicos ou em criatórios comerciais. No entanto, são escassas as informações sobre vários aspectos da criação dessas aves, inclusive sobre a infecção por Isospora spp., que pode se manifestar com curso agudo e presença de emagrecimento, má-absorção e má-digestão, devido à lise de enterócitos ${ }^{1}$, além de alta mortalidade $^{2,3}$.

Há diversos estudos sobre a intensidade e periodicidade de eliminação de oocistos de Isospora spp. em aves de vida livre, que visam principalmente à determinação do padrão diurno e/ou diário da eliminação de oocistos de forma intermitente em aves infectadas naturalmente ou experimentalmente ${ }^{4-11}$. No entanto, não há estudos relacionados à presença de oocistos de Isospora spp., por períodos prolongados, em fezes de aves criadas em cativeiro.

Aves de vida livre desenvolvem infecção crônica e permanecem portadoras do parasito, mesmo após utilização de medicamentos anticoccidianos, e não há desenvolvimento de imunidade específica para prevenção de infecção aguda após desafio posterior com oocistos da mesma espécie de Isospora ${ }^{12}$.

É evidente a carência de informações técnicas sobre a ocorrência e métodos de controle relacionados às enfermidades de aves criadas em cativeiro, onde se observa que na prevenção e tratamento de enfermidades infecciosas e parasitárias predomina o empirismo, com administração de drogas de forma totalmente aleatória e sem nenhum embasamento científico, o que pode levar à utilização inadequada de medicamentos anticoccidianos, com ocorrência de quadros de intoxicação ou desenvolvimento de resistência dos coccídios às drogas utilizadas, como observado com frequência na eimeriose aviária ${ }^{13}$.

O presente estudo teve como objetivo pesquisar, em longo prazo, a presença de oocistos de Isospora spp. em várias espécies de aves da ordem Passeriformes, naturalmente infectadas. into account not only the presence of the parasite in feces, but also the intensity of oocysts shedding, as well as evaluation of sanitary and nutritional management and the presence of clinical signs and/or mortality.

\section{MATERIAL E MÉTODOS}

Foram coletadas 289 amostras de fezes de 25 aves (Tabela 1), em dois criatórios onde houve comprovação prévia de infecção por Isospora, nos quais havia alojamento de curiós (Oryzoborus angolensis), sabiás-laranjeira (Turdus rufiventris), pintassilgos-da-Venezuela (Carduelis cucullatus) e pintassilgos-do-nordeste (Carduelis yarrellii) (criatório 1) e de curiós e bicudos (Oryzoborus maximiliani) (criatório 2). O número de aves selecionadas para coleta foi de 10 a $100 \%$, em relação ao total de aves por espécie presente na criação, variando de uma a seis aves de cada espécie. As aves estavam alojadas em gaiolas individuais, que foram identificadas para permitir a coleta seriada de amostras fecais da mesma ave.

Foi realizada coleta individual, em aves adultas (após o início do período reprodutivo), com periodicidade mensal, por um período de 13 meses, com exceção dos meses de abril e agosto, devido à indisponibilidade do proprietário do criatório 1 . Apesar de a eliminação da maioria dos oocistos de Isospora ocorrer no período que compreende as últimas horas da tarde e as primeiras horas da noite $^{5,7,9}$, devido à disponibilidade dos proprietários, as amostras foram constituídas por fezes eliminadas durante $24 \mathrm{~h}$, e foram colhidas no início da manhã, em gaiolas forradas com forro de papel, que era substituído a cada colheita, e foram armazenadas em solução de bicromato de potássio a 2,5\%, inicialmente por $96 \mathrm{~h}$, em temperatura ambiente, em recipiente aberto, para que ocorresse a esporulação de oocistos. Posteriormente foram armazenadas a $4^{\circ}$ C.

As amostras de fezes foram homogeneizadas e coadas em peneiras de plástico descartáveis e submetidas à lavagem por centrifugação com água deionizada/tween $20,0,01 \%$, seguida da purificação e concentração de oocistos utilizando-se o método de centrífugo-flutuação em solução de Sheather. Dois mililitros do sobrenadante da solução de Sheather foram diluídos em água deionizada/tween 20, 0,01\%, e submetidos à centrifugação com água deionizada/tween $20,0,01 \%$. No sedimento 
resultante do processo de purificação, foi pesquisada a presença de oocistos de Isospora spp. por meio da técnica de coloração negativa com verde malaquita ${ }^{14}$. Essa técnica, apesar de desenvolvida originalmente para visualização de oocistos de Cryptosporidium spp., permite excelente visualização de oocistos de Isospora spp.

A identificação morfológica de oocistos de Isospora spp. ${ }^{15}$ foi realizada com utilização de objetiva de $40 \mathrm{X}$ e $100 \mathrm{X}$. A quantificação da eliminação de oocistos foi realizada utilizando-se um método semiquantitativo, descrito a seguir: negativo (-); escore 1: 1 a 20 oocistos por lâmina; escore 2: 21 a 50 oocistos por lâmina; escore 3: mais de 1 oocisto por campo em objetiva de $20 \mathrm{X}$; escore 4: mais de 10 oocistos por campo em objetiva de $20 \mathrm{X}$. Devido ao grande número de amostras e de espécies estudadas, as características morfológicas e morfométricas dos oocistos não foram analisadas.

Para análise estatística dos escores de eliminação de oocistos, nos diferentes meses de colheita, foram empregados os testes não paramétrico de Kruskal-Wallis e de Dunn, a 5\% de probalilidade, utilizando-se o software Biostat versão 5.0.

Este trabalho foi desenvolvido após aprovação do Comitê de Ética em Experimentação Animal, processo número 14/06, UNESP, Campus de Araçatuba.

\section{RESULTADOS E DISCUSSÃO}

$\mathrm{Na}$ maioria das aves de todas as espécies analisadas foi observada eliminação de oocistos em pequena quantidade (escore 1), de forma intermitente e por períodos prolongados (Tabela 1). Das 289 amostras, $159(55,02 \%)$ apresentaram positividade para oocistos de Isospora (Figura 1) e $130(44,98 \%)$ foram negativas. Todas as aves apresentaram oocistos de Isospora pelo menos uma vez no período de 13 meses, o que implica em manutenção constante desse coccídio nos criatórios, durante todo o ano. Não há estudos sobre o padrão de eliminação de oocistos em aves mantidas em cativeiro e em diferentes períodos do ano. As pesquisas relacionadas à eliminação de oocistos de Isospora referem-se às aves de vida livre, e descrevem variação na quantidade diária de oocistos eliminados em fezes, assim como maior eliminação no período da tarde, particularmente no final da tarde e início da noite $5,7,10,16,17$.

Em aves Passeriformes de vida livre, na região centro-sul do Brasil, o período reprodutivo inicia-se em agosto e é mais comum entre outubro e janeiro, com pico em novembro, enquanto que a muda de penas tem início em outubro e é mais comum entre dezembro em abril, com pico em fevereiro $^{18}$. De acordo com informações colhidas dos proprietários dos criatórios utilizados neste experimento, em todas as espécies de aves analisadas o período reprodutivo compreende os meses de setembro a março, seguido do período de muda das penas.

Pela análise dos escores de eliminação de oocistos (Tabela 1), observa-se que há maior eliminação nos meses correspondentes ao início e pico do período reprodutivo. No entanto, foi observado aumento na eliminação de oocistos no mês de março, que corresponde ao final do período reprodutivo e início do período de mudas das penas, e no mês de julho, provavelmente pelo estresse resultante de baixas temperaturas ou, ainda, por alterações hormonais que antecedem o início do período reprodutivo, em agosto.

Várias espécies de Isospora podem infectar uma mesma espécie de ave, como descrito em $O$. angolensis e $S$. caerulescens ${ }^{19,20 .}$

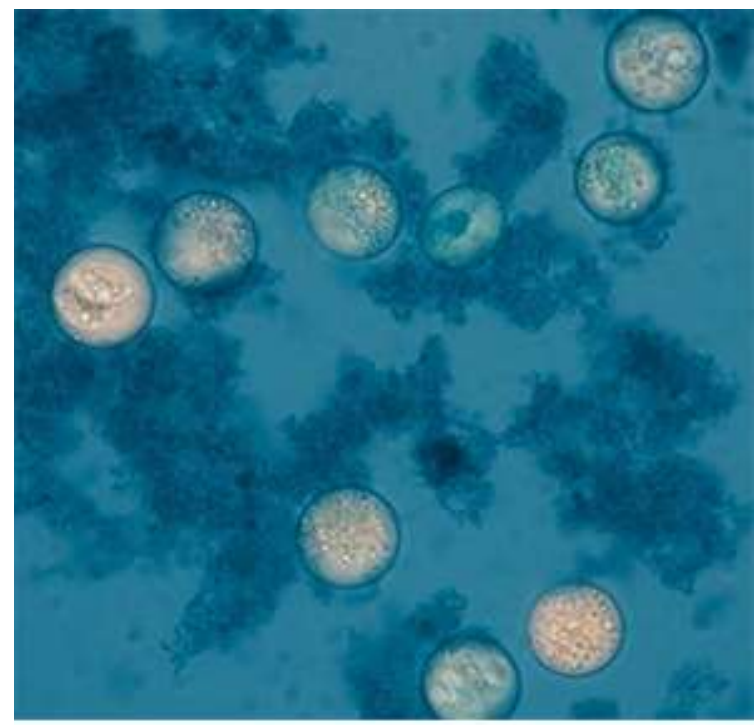

Figura 1. Fotomicrografia de oocistos de Isospora sp. em fezes de Oryzoborus maximiliani. Coloração negativa com verde malaquita (400x).

Não há descrição das espécies de Isospora que infectam bicudos ou pintassilgos ${ }^{21}$; no entanto, em sabiás há descrição de cinco espécies desse coccídio $^{21,22}$. Apesar de a identificação da espécie de Isospora não ter sido realizada neste experimento, há possibilidade de que em uma mesma ave, no mesmo período de colheita, houvesse a presença de mais de uma espécie do parasito e em diferentes fases de infecção, conforme sugerido por Filipiak et al. ${ }^{7}$. 
Tabela 1. Escore de eliminação de oocistos de Isospora spp. em aves adultas

\begin{tabular}{|c|c|c|c|c|c|c|c|c|c|c|c|c|c|c|c|c|}
\hline \multirow[b]{2}{*}{ Criatório } & \multirow[b]{2}{*}{ Nome Comum } & \multirow[b]{2}{*}{ Espécie } & \multirow[b]{2}{*}{ ave } & \multicolumn{5}{|c|}{2007} & \multicolumn{8}{|c|}{2008} \\
\hline & & & & set & out & nov & dez & jan & fev & mar & $\mathrm{abr}$ & mai & jun & jul & ago & set \\
\hline \multirow{18}{*}{1} & \multirow{3}{*}{$\begin{array}{l}\text { Pintassilgo do } \\
\text { Nordeste }\end{array}$} & \multirow{3}{*}{$\begin{array}{l}\text { Carduelis } \\
\text { yarrellii }\end{array}$} & A & 1 & 1 & 1 & 1 & - & 1 & 1 & $*$ & - & 1 & 1 & $*$ & 1 \\
\hline & & & B & 1 & 1 & 1 & 1 & - & - & 1 & $*$ & - & 1 & 1 & * & 1 \\
\hline & & & $\mathrm{C}$ & 1 & 2 & 1 & - & - & 1 & - & $*$ & - & - & 1 & $*$ & 1 \\
\hline & \multirow{3}{*}{$\begin{array}{l}\text { Pintassilgo da } \\
\text { Venezuela }\end{array}$} & \multirow{3}{*}{$\begin{array}{l}\text { Carduelis } \\
\text { cucullatus }\end{array}$} & A & 1 & 1 & 1 & - & 1 & - & - & $*$ & - & 1 & 1 & $*$ & 1 \\
\hline & & & B & 1 & - & 1 & - & 1 & - & - & $*$ & 1 & - & - & * & - \\
\hline & & & $\mathrm{C}$ & 1 & 1 & 1 & - & 1 & - & - & $*$ & - & - & - & $*$ & 1 \\
\hline & \multirow{3}{*}{$\begin{array}{c}\text { Sabiá } \\
\text { Laranjeira }\end{array}$} & \multirow{3}{*}{$\begin{array}{l}\text { Turdus } \\
\text { rufiventris }\end{array}$} & A & 1 & 1 & 1 & 1 & - & 1 & - & $*$ & 1 & - & 1 & $*$ & 1 \\
\hline & & & $\mathrm{B}$ & 1 & 1 & 1 & 1 & - & - & 1 & $*$ & - & 1 & 1 & * & 1 \\
\hline & & & $\mathrm{C}$ & 1 & 1 & 1 & 1 & - & - & - & $*$ & - & - & 1 & $*$ & 1 \\
\hline & \multirow{3}{*}{ Curió } & \multirow{3}{*}{$\begin{array}{l}\text { Oryzoborus } \\
\text { angolensis }\end{array}$} & A & 1 & 1 & 1 & - & - & - & - & $*$ & - & - & 1 & $*$ & 1 \\
\hline & & & B & 1 & 1 & 2 & 1 & - & - & - & $*$ & 1 & - & 1 & $*$ & 2 \\
\hline & & & $\mathrm{C}$ & 1 & 1 & 1 & - & - & - & 1 & $*$ & 1 & - & 1 & $*$ & 1 \\
\hline & \multirow{6}{*}{ Bicudo } & \multirow{6}{*}{$\begin{array}{l}\text { Oryzoborus } \\
\text { maximiliani }\end{array}$} & A & 2 & 2 & 1 & 1 & 1 & - & - & $*$ & 1 & - & 2 & $*$ & 1 \\
\hline & & & B & 1 & 1 & 2 & - & - & 1 & - & $*$ & - & - & 1 & $*$ & 2 \\
\hline & & & $\mathrm{C}$ & 1 & 1 & 1 & - & 1 & 1 & 1 & $*$ & - & 1 & 1 & $*$ & 1 \\
\hline & & & $\mathrm{D}$ & 1 & 1 & 1 & - & 1 & - & 1 & $*$ & - & - & 1 & $*$ & 1 \\
\hline & & & E & 1 & 1 & 1 & 1 & - & 1 & 1 & $*$ & - & 1 & 1 & $*$ & 1 \\
\hline & & & $\mathrm{F}$ & 1 & 1 & 2 & 1 & - & - & - & $*$ & - & - & 1 & $*$ & 1 \\
\hline \multirow{7}{*}{2} & \multirow{3}{*}{ Curió } & \multirow{3}{*}{$\begin{array}{l}\text { Oryzoborus } \\
\text { angolensis }\end{array}$} & A & 1 & - & 1 & 1 & - & - & 1 & - & - & 1 & 1 & - & 1 \\
\hline & & & B & - & - & - & - & - & - & 1 & - & - & - & 1 & - & - \\
\hline & & & $\mathrm{C}$ & - & - & 1 & - & - & - & 1 & 1 & 1 & - & - & - & 1 \\
\hline & \multirow{4}{*}{ Bicudo } & \multirow{4}{*}{$\begin{array}{l}\text { Oryzoborus } \\
\text { maximiliani }\end{array}$} & A & - & 1 & - & - & - & - & - & - & - & - & - & 1 & - \\
\hline & & & B & 1 & 1 & 1 & - & - & - & 1 & 1 & 1 & - & 1 & 1 & 1 \\
\hline & & & $\mathrm{C}$ & 1 & 1 & - & - & - & - & - & 1 & - & - & - & 1 & 1 \\
\hline & & & $\mathrm{D}$ & - & 1 & - & - & - & - & 1 & 1 & - & - & - & - & 1 \\
\hline \multicolumn{4}{|c|}{ Média dos escores de eliminação de oocistos } & $0,9^{\mathrm{a}}$ & $0,9^{\mathrm{a}}$ & $1,0^{\mathrm{a}}$ & $0,4^{\mathrm{ab}}$ & $0,3^{\mathrm{b}}$ & $0,3^{\mathrm{b}}$ & $0,5^{\mathrm{ab}}$ & $* *$ & $0,3^{\mathrm{bc}}$ & $0,3^{\mathrm{bc}}$ & $0,8^{\mathrm{ac}}$ & $* *$ & $1,0^{\mathrm{a}}$ \\
\hline
\end{tabular}

Apesar de estarem infectadas por Isospora, as aves analisadas não apresentaram sinais clínicos de isosporose. Duas aves do criatório 1 ( $O$. angolensis e $O$. maximiliani), que não estavam entre as aves selecionadas para colheita de fezes, vieram a óbito em decorrência de isosporose aguda, diagnosticada por meio de exame citológico e histopatológico. Como observado comumente em Passeriformes criados em cativeiro, há episódios esporádicos de infecção aguda, em algumas situações com alto índice de mortalidade, em decorrência do fato de que os animais previamente infectados e que apresentam infecção crônica, quando são desafiados com oocistos da mesma espécie de Isospora, podem apresentar sinais clínicos e mortalidade aguda ${ }^{1}$. Da mesma forma, diversas causas de imunossupressão, incluindo alterações fisiológicas nos períodos reprodutivo e de muda das penas, má nutrição, deficiências no manejo ou presença de infecções concomitantes podem 
predispor a recidivas e ao surgimento de quadro clínico agudo ${ }^{1,23,24}$, o que provavelmente ocorreu com as duas aves do criatório 1 .

Os resultados observados neste experimento fornecem subsídios para o controle da isosporose em passeriformes criados em cativeiro; as decisões sobre a realização de tratamento profilático ou curativo, assim como sobre medidas higiênico-sanitárias a serem adotadas devem levar em consideração não somente a presença do parasito em fezes, mas também a intensidade de eliminação de oocistos, assim como a avaliação do manejo higiênico sanitário e nutricional e a presença de sinais clínicos e/ou de mortalidade.

\section{AGRADECIMENTOS}

À Fundação de Amparo à Pesquisa do Estado de São Paulo (FAPESP), pelo apoio financeiro (processo n. 2007/54312-2).

\section{REFERÊNCIAS}

1. Hõrak P, Saks L, Karu U, Ots I, Surai PF, McGraw KJ. How coccidian parasites affect health and appearance of greenfinches. Journal of Animal Ecology. 2004; 73(5): 935-47.

2. Sironi G. Concurrent calicivirus and Isospora lacazei infections in goldfinches (Carduelis carduelis). Veterinary Record. 1994; 134(8):196.

3. Giacomo R, Stefania P, Ennio T, Giorgina BC, Giovanni B, Giacomo R. Mortality in black siskins (Carduelis atrata) with systemic coccidiosis. Journal of Wildlife Diseases. 1997; 33(1):152-57.

4. Zinke A, Schnebel B, Dierschke V, Ryll M. Prevalence and intensity of excretion of coccidial oocysts in migrating passerines on Helgoland. Journal of Ornithology. 2004; 145(1):74-8.

5. Dolnik O. The relative stability of chronic Isospora Sylvianthina (Protozoa: Apicomplexa) infection in blackcaps (Sylvia atricapilla): evaluation of a simplified method of estimating isosporan infection intensity in passerine birds. Parasitology Research. 2006; 100(1):15560 .

6. López G, Figuerola J, Soriguer R. Time of day, age and feeding habits influence coccidian oocyst shedding in wild passerines. International Journal for Parasitology. 2007; 37(1):559-64.

7. Filipiak L, Mathieu F, Moreau J. Caution on the assessment of intestinal parasitic load in studying parasitemediated sexual selection: The case of blackbirds coccidiosis. International Journal for Parasitology. 2009; 39(6):741-46.

8. Dolnik O.V, Dolnik V.R, Bairlein F. The effect of host foraging ecology on the prevalence and intensity of coccidian infection in wild passerine birds. Ardea. 2010; 98(1):97-103.

9. Dolnik OV, Metzger BJ, Loonen MJJE. Keeping the clock set under the midnight sun: diurnal periodicity and synchrony of avian Isospora parasites cycle in the High Arctic. Parasitology. 2011; 138(9):1077-81.

10. Coelho CD, Berto BP, Neves DM, Oliveira VM, Flausino W, Lopes CWG. Oocyst shedding by greenwinged-saltator (Saltator similis) in the diagnostic of coccidiosis and Isospora similisi n. sp.(Apicomplexa: Eimeriidae). Brazilian Journal of Veterinary Parasitology. 2013; 22(1):64-70.

11. Vasconcellos MSD, Batista LCSO, Vidal LGP, Passos MM. Intensidade de infecção por Isospora spp. (Apicomplexa: Eimeriidae) em trinca-ferros-verdadeiros Saltator similis d'Orbigny, Lafresnaye (Passeriformes: Cardinalidae) mantidos em cativeiro no Município de Valença, Estado do Rio de Janeiro, Brasil. Coccidia. 2013; 1(2):39-43.

12. Hõrak P, Saks L, Karu U, Ots I. Host resistance and parasite virulence in greenfinch coccidiosis. Journal of Evolutionary Biology. 2006; 19(1):277-88.

13. Peek HW, Landman WJ. Coccidiosis in poultry: anticoccidial products, vaccines and other prevention strategies. Veterinary Quaterly. 2011; 31(3):143-61.

14. Elliot A, Morgan UM, Thompsom RCA. Improved staining method for detecting Cryptosporidium oocysts in stools using malachite green. The Journal of General and Applied Microbiology. 1999; 45(3):139-42.

15. Berto BP, Mcintosh D, Lopes CWG. Studies on coccidian oocysts (Apicomplexa: Eucoccidiorida). Brazilian Journal of Veterinary Parasitology. 2014; 23(1):1-15.

16. Misof K. Diurnal cycle of Isospora spp. oocyst shedding in Eurasian blackbirds (Turdus merula). Canadian Journal of Zoology. 2004; 82(5):764-768.

17. Martinaud G, Billaudelle M, Moreau J. Circadian variation in shedding of the oocysts of Isospora turdi (Apicomplexa) in blackbirds (Turdus merula): An adaptative trait against desiccation and ultraviolet radiation. International Journal for Parasitology. 2009; 39(6):735-39.

18. Marini MA, Durães R. Annual patterns of molt and reproductive activity of passerines in south-central Brazil. The Condor. 2001; 103(4):767-75.

19. Carvalho Filho PR, Meireles GS, Ribeiro CT, Lopes CWG. Three new species of Isospora Schneider, 1881 (Apicomplexa: Eimeridae) from the double-collared seed eater, Sporophila caerulescens (Passeriformes: Emberizidae), from Eastern Brazil. Memórias do Instituto Oswaldo Cruz. 2005; 100(2):151-54.

20. Silva EAT, Literák I, Koudela B. Three new species of Isospora Schneider, 1881 (Apicomplexa:Eimeriidae) from the lesser seed-finch, Oryzoborus angolensis (Passeriformes:Emberezidae) from Brazil. Memórias do 
Instituto Oswaldo Cruz. 2006; 101(5): 573-76.

21. Berto BP, Flausino W, Luz HR, Ferreira I, Lopes CWG. Coccidia of New World passerine bir $\neg$ ds (Aves: Passeriformes): a review of Eimeria Schneider, 1875 and Isospora Schneider, 1881 (Apicomplexa: Eimeriidae). Systematic Parasitology. 2011; 80(3):159-204.

22. Lopes BB, Berto BP, Luz HR, Galvão GD, Ferreira I, Lopes CWG. Isospora massardi sp. nov. (Apicomplexa: Eimeriidae) from the white-necked thrush Turdus albicollis (Passeriformes: Turdidae) from Brazil. Acta Parasitologica. 2014; 59(2):272-75

23. Davis GS. The effects of long-term caging and molt of Single Comb White Leghorn hens on heterophil to lymphocyte ratios, corticosterone, and thyroid hormones. Poultry Science. 2000; 79(4):514-18.

24. Cox FEG. Concomitant infections, parasites and immune responses. Parasitology. 2001; 122(1):S23-S38.

Protocolado em: 14 fev. 2013. Aceito em 08 jul. 2014 